\title{
Intensity noise characteristics of multimode VCSELs
}

\author{
A. Quirce, A. Valle, C. Giménez, and L. Pesquera
}

\begin{abstract}
The intensity noise spectra of several multitransverse mode $1550-\mathrm{nm}$ vertical-cavity surface-emitting lasers (VCSELs) have been experimentally investigated. For a VCSEL emitting in two parallel polarized transverse modes the noise spectra of the individual modes and total power show two resonance peaks. The frequencies at which both peaks appear have been measured as a function of the bias current. Our experimental results confirm the theoretical predictions of $A$. Valle et al, IEEE J. Quantum Electron, vol. 40, 597, 2004. Differences in the noise spectra are experimentally observed when considering a VCSEL emitting in three transverse modes with polarization instabilities. We show that additional peaks appear in the noise spectra of the total power as the bias current is increased.
\end{abstract}

Index Terms - Semiconductor lasers, Vertical-cavity surfaceemitting lasers (VCSELs), transverse modes, relative intensity noise (RIN), mode partition noise (MPN).

\section{INTRODUCTION}

$\mathrm{V}$ ertical-cavity surface-emitting lasers (VCSELs) are the most common light emitters for high-speed short-distance data communications at $850 \mathrm{~nm}$ over multimode optical fibers. [1-2]. VCSELs usually have large active diameters allowing for multiple transverse modes. Multitransverse mode operation ensures low source coherence, greatly minimizing interference effects like the modal noise-induced bit error rate in multimode fiber systems [3]. 1550-nm multimode VCSELs are also of interest for use in the fiber metropolitan area network because of their low cost [4]. The emission in multiple transverse modes can be attributed to "spatial hole burning" effects [5]. The polarization of the light emitted by the VCSELs is not as well stabilized as in edge-emitting lasers and VCSELs are known to have a number of polarization instabilities. Several experimental studies show that the emission in several transverse and polarization modes affects quantities like the relative intensity noise (RIN) [6-13] and the small-signal modulation response [14] of VCSELs. A better

Manuscript received June, 2010. This work has been funded in part by the Ministerio de Ciencia e Innovación, Spain, under project TEC2009-14581C02-02.

A. Quirce, A. Valle, C. Giménez, and L. Pesquera are with the Instituto de Física de Cantabria, Consejo Superior de Investigaciones Científicas (CSIC)Universidad de Cantabria, E-39005 Santander, Spain. A. Quirce is also with the Departamento de Física Moderna, Univ. de Cantabria, Facultad de Ciencias, E-39005, Santander, Spain (Phone: (34) 942 201465. Fax: (34) 942 200935. E-mail: quirce@ifca.unican.es; valle@ifca.unican.es; pesquerl@ifca.unican.es).

Copyright (c) 2011 IEEE understanding of those quantities is important for applications in optical communications systems and optical interconnects [6].

Theoretical investigations of RIN in linearly polarized multimode VCSELs have shown that the RIN spectra of individual transverse modes [6],[15-17] and of the total power [16-17] have several maxima. Experiments have demonstrated that filtered RIN spectra have multiple resonance peaks [6]. Also experimental RIN spectra of the total power have displayed several peaks [11]. Localized increases of RIN at low frequencies as a function of the bias current have also been experimentally observed [7], [10-11] in good agreement with the theory [16-17]. Theoretical modelling of RIN in parallel polarized multimode VCSELs has been performed by using spatially independent [6] and spatially dependent [15-17] stochastic rate equations. Analytical expressions of the RIN obtained with the previous spatially dependent models show that there are two resonance peaks in the noise spectra of the individual modes and total power of a two-transverse mode VCSEL [17]. Those peaks appear at frequencies that correspond to the relaxation oscillation frequencies of the multimode laser [17]. The dependence of the frequencies at which RIN peaks appear on the bias current applied to the VCSEL has also been obtained theoretically [17]. Although some experimental results show multipleresonance peaks in the RIN of individual modes [6] and of the total power [11] there are no systematic experimental investigations of the dependence of RIN spectra of multimode VCSELs on their applied bias current.

In this work we present experimental results of intensity noise spectra obtained for several commercially available 1550-nm multimode VCSELs. We analyze the dependence of those spectra on the bias current applied to the VCSEL. The first VCSEL is characterized by emission in two transverse modes with the same linear polarization over a very wide bias current range. In this way we perform a comparison with the theoretical results corresponding to a VCSEL emitting in two parallel polarized transverse modes [17]. Two resonance peaks appear in the noise spectra of the individual modes and total power of the VCSEL in agreement with [17]. We have measured the frequencies corresponding to both peaks as a function of the bias current. We have obtained a similar dependence to the one found in [17]. We have also extended our investigations to two more VCSELs that are characterized by emission in three transverse modes with polarization instabilities for large values of the bias current. Similar results to those obtained with the first VCSEL are obtained for 
currents in which there is emission in one or two transverse modes. The situation changes for larger values of the current because the simultaneous presence of three transverse modes with polarization instabilities gives rise to a change of the structure of the intensity noise spectra characterized by the appearance of a set of additional peaks.

The contribution is structured as follows. After this short introduction we describe our experimental setup in section II. In section III we present the results corresponding to the twotransverse mode VCSEL. In section IV the experimental results corresponding to two three-transverse mode VCSELs with polarization instabilities are shown. We close with a discussion and a summary in section $\mathrm{V}$.

\section{EXPERIMENTAL SETUP}

The measurements were performed on two different quantum-well commercial VCSELs $\left(\right.$ Raycan $^{\mathrm{TM}}$ ) that emit around $1560 \mathrm{~nm}$. The bias current and temperature were controlled by a laser driver (Thorlabs LDC200) and a temperature controller (Thorlabs TED200), respectively. Temperature was held constant at $297 \mathrm{~K}$ during the experiments. Spectral measurements were performed by using an Optical Spectrum Analyzer (OSA, Anritsu MS9710B), a Fabry-Perot (FP) Optical Spectrum Analyzer (Micron Optics FFP-TF2) and a RF-spectrum analyzer (Anritsu MS2719B). The output of the VCSEL was coupled into an optical isolator after which a 50/50 coupler split the light in two detection arms. In the first arm an amplified photodetector (Thorlabs PDA8GS) and the RF-spectrum analyzer were used to obtain the noise spectra. In the second arm the optical spectra were measured by using the OSA or the FP analyzer.

Fig. 1 shows the optical spectrum of the first VCSEL (VCSEL 1) obtained by the OSA at two different bias currents, $I_{V C S E L}$. The aperture diameter of the VCSEL is around $12 \mu \mathrm{m}$._For small values of the bias current the VCSEL emits in a single transverse mode with a wavelength near $1560 \mathrm{~nm}$, as it is shown in Fig. 1(a). The two peaks that appear at longer wavelengths correspond to the two orthogonal linear polarizations of the fundamental transverse mode. We have checked that both peaks correspond to orthogonal linear polarizations by using a polarization beam splitter. The reason of the polarization splitting of the transverse modes is the strong birefringence due to the strain of the material introduced during manufacturing of the VCSEL. The lowest of those peaks $(1560.5 \mathrm{~nm})$ corresponds to the depressed polarization (the so-called orthogonal polarization) of the fundamental transverse mode. The dominant linear polarization (the so-called parallel polarization) of the fundamental mode appears at a shorter wavelength (1559.95 $\mathrm{nm})$. This VCSEL is characterized by a large value of the birefringence parameter because the frequency difference between both linear polarizations is $68.8 \mathrm{GHz}$. The peaks that appear at shorter wavelengths correspond to non lasing higherorder transverse modes. For larger values of the bias current the VCSEL emits also in a higher-order transverse mode as it can be seen in Fig. 1(b). The higher-order mode appears at a wavelength of $1560.61 \mathrm{~nm}$ with a linear polarization that is parallel to that of the fundamental mode. We have checked that the polarizations of both transverse modes are parallel by using a polarization beam splitter. The frequency difference between the parallel polarized fundamental and higher-order transverse mode is around $267.5 \mathrm{GHz}$. That frequency separation is large enough to separate the contributions of each transverse mode to the total power with a bandpass tunable filter (Newport TBF-1550). In this way the transverse-mode resolved Light-current characteristics of the VCSEL 1 can be obtained.
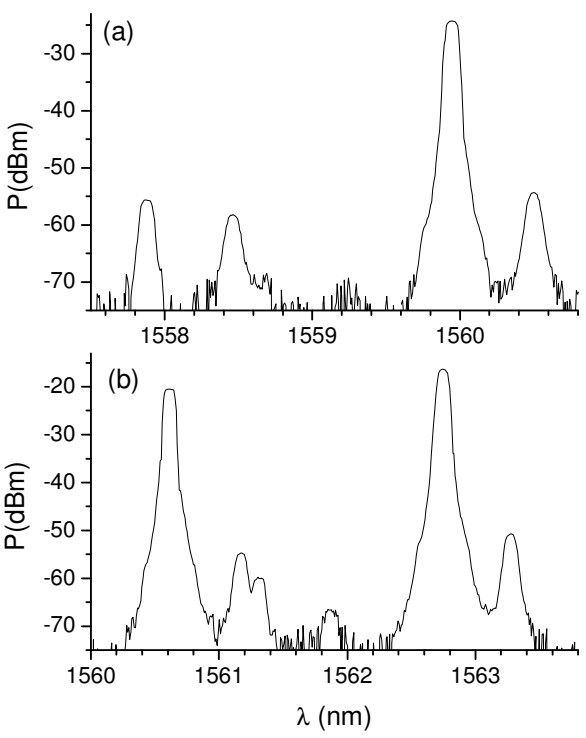

Fig. 1. Optical spectrum of VCSEL 1 when the applied bias current is (a) $3.51 \mathrm{~mA}$ and (b) $9.80 \mathrm{~mA}$

Fig. 2 shows the power of the transverse modes and the total power as a function of the bias current. Only two transverse modes contribute to the total power in an appreciable way. Both modes have the parallel polarization over the whole bias current range. In this way the VCSEL 1 is an example of a linearly polarized two-transverse mode VCSEL whose behavior was described in a theoretical work [17]. We also observe in Fig. 2 that the power of each transverse mode depends linearly on the bias current. The slopes of these straight lines change when the new transverse mode appears at $3.9 \mathrm{~mA}$. That value correspond to 1.38 times the threshold current, $\mathrm{I}_{\text {th }} \quad\left(\mathrm{I}_{\text {th }}=2.83 \mathrm{~mA}\right)$. For bias currents smaller than 7.5 $\mathrm{mA}$ those observations are in agreement with a previous theoretical work that described analytically the transverse mode resolved L-I characteristics of weakly-index guided multimode VCSELs [18]. The disagreement with the theoretical predictions when the bias current is larger than 7.5 $\mathrm{mA}$ could be attributed to the fact that the theoretical hypothesis of fixed transverse modal profiles is no longer fulfilled at those large values of the current. 


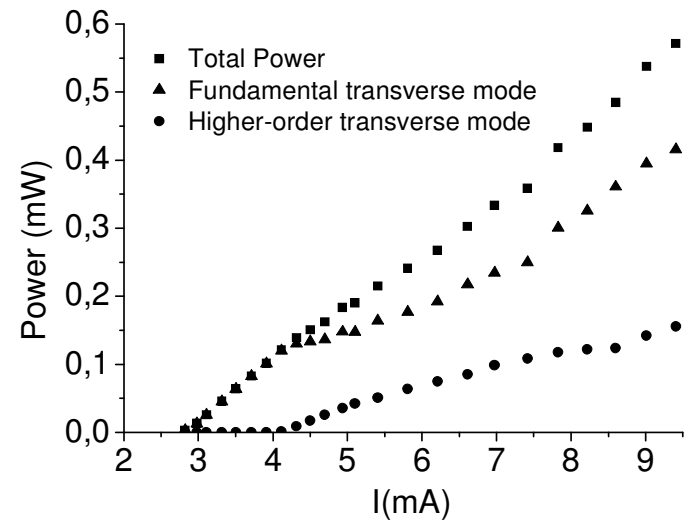

Fig. 2. Power of the transverse modes and total power of VCSEL 1 as a function of the bias current.

\section{INTENSITY NOISE SPECTRA OF PARALLEL POLARIZED TWO-MODE VCSEL}

In this section we analyze the intensity noise spectra of the individual modes and total power as a function of the bias current applied to the parallel polarized two mode VCSEL 1. Fig. 3 shows the spectra (thin lines) of the total power, obtained in the RF spectrum analyzer, for six different values of the bias current, I. There are many peaks separated by a constant frequency difference of around $100 \mathrm{MHz}$ in all the spectra. That frequency difference corresponds to the reflections that occur at the end of the 1 meter fiber that is pigtailed to the VCSEL. The underlying structures of those peaks have been obtained by performing the Savitzky-Golay smoothing algorithm. The smoothed spectra are also plotted in Fig. 3 with thick red lines. Fig. 3(a) shows that when the VCSEL emits in the fundamental transverse mode there is only one clear peak in the smoothed spectra at $0.9 \mathrm{GHz}$ (a much weaker harmonic peak appears at $1.8 \mathrm{GHz}$ ). The frequency at which the peak appears increases as the bias current increases as it can be seen in Fig. 3(b). Harmonics of the main peak appear because the power fluctuations have an underlying periodic structure. Fig. 3(c) shows that the excitation with significant power of the higher-order transverse mode (see also Fig. 2) is accompanied by the emergence of a second peak at a lower frequency $(0.75 \mathrm{GHz})$. The bump that appears at 1.5 $\mathrm{GHz}$ corresponds to the first harmonic of that peak. In a similar way Fig. 3(d)-(f) show that two clear peaks appear in the smoothed spectrum for larger values of I for which only two transverse modes are excited. Further small peaks also appear at larger frequencies in Fig. 3(d)-(f) that correspond to sum-frequency of the lower frequency peaks. Fig. 3 then shows that two resonance peaks appear in the spectra of the total power of a VCSEL that emits in two parallel transverse modes in agreement with previous theoretical studies [17].

The intensity noise spectra that correspond to individual transverse modes were also measured by inserting the tunable bandpass filter before the RF-spectrum analyzer. Fig. 4 shows the intensity noise spectrum of the fundamental, higher order transverse mode and of the total power for different values of the bias current. As in Fig. 3 the non smoothed and smoothed data are plotted with thin and thick solid lines, respectively. Fig. 4 shows that in the two-transverse mode regime the spectra of the individual transverse modes have also two peaks that appear at the same frequencies than those corresponding to both peaks of the spectrum of the total power. This result is also in agreement with previous theoretical analysis [17]. RIN spectra of individual modes are usually higher than that of the total power [16-17]. However Fig. 4 shows that RF spectra of individual modes are smaller than that of the total power. This can be explained by the way of measuring our RF spectra. A tunable filter is placed before the RF-spectrum analyzer to measure RF spectra of individual modes in contrast to the measurement of the RF spectra of the total power in which no filter is used. The tunable filter produces a significant loss of power that lowers RF spectra of individual modes below that of the total power. We also note that the RIN is obtained from the RF spectra by normalizing with the square of the averaged power [16-17] and therefore they are not the same quantities.
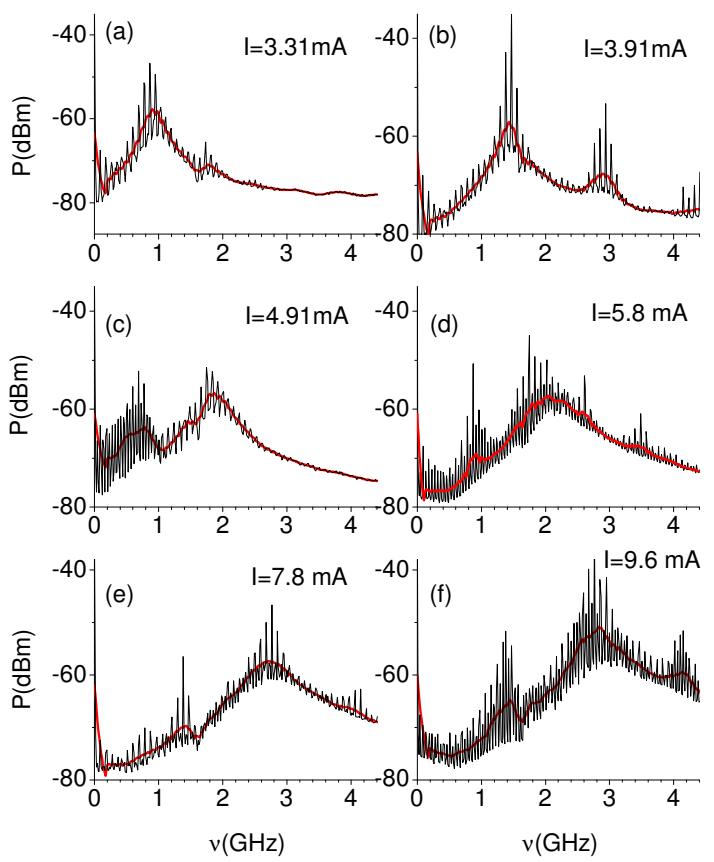

Fig. 3. (color online) RF spectra of the total power of the two-transverse mode VCSEL at different values of the bias current. The smoothed spectra are plotted with thick (red) lines.

We showed in Fig. 3 that the frequencies at which the spectrum of the total power has a maximum increase as the bias current is increased. We show in Fig. 5(a) the detailed dependence of those frequencies on the bias current. We have plotted the frequencies obtained with the smoothed spectra. For currents smaller than $3.9 \mathrm{~mA}$ the VCSEL is single transverse mode and the noise spectrum has only one maximum. The typical linear relationship between the square of that frequency and $\mathrm{I}-\mathrm{I}_{\mathrm{th}}$ is obtained in the single mode regime as illustrated in Fig. 5 (b). When the laser begins to emit in the two transverse modes the spectra develops two 
maxima as it is shown in Fig. 5(a). This situation is maintained for larger values of the bias current. Both frequencies at which the noise spectra has a maximum increase when increasing the current until saturating when $\mathrm{I}>7.5 \mathrm{~mA}$. The theoretical results of Ref. [17] show similar trends to the observed in our experiment while $\mathrm{I}<7.5 \mathrm{~mA}$. We have performed a detailed analysis of the frequency versus current dependence. The dependence of the frequency of the second peak on the current is such that $\mathrm{V} \sim\left(\mathrm{I}-\mathrm{I}_{\mathrm{th}, \mathrm{h}}\right)^{0.6}$ where $\mathrm{I}_{\mathrm{th}, \mathrm{h}}$ is the threshold current of the high-order mode. The theoretical results of Fig. 6 of Ref. [17], obtained for a VCSEL with half the diameter of our VCSEL, are such that $\mathrm{V} \sim\left(\mathrm{I}-\mathrm{I}_{\mathrm{th}, \mathrm{h}}\right)^{0.52}$. We note that the range of bias currents at which the theoretical predictions fail (I $>7.5$ $\mathrm{mA}$ ) is similar, in particular for the current-dependence of the power of individual transverse modes and that of the frequencies at which the spectrum has a maximum. We believe that in this bias current range the failure of the theory for describing the experimental results can be related to the theoretical assumption of fixed transverse modal profiles that could not be the case for large values of I as it was already discussed in relation to Fig. 2.

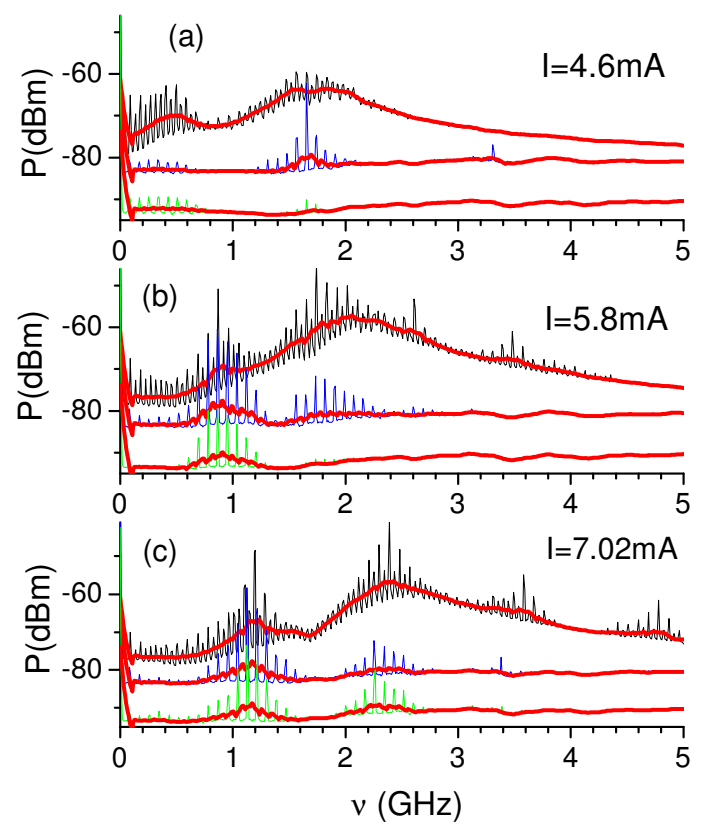

Fig. 4. (color online) RF spectra of the individual transverse modes and of the total power of the two-transverse mode VCSEL at different values of the bias current. Upper (black), middle (blue) and lower (green) curves correspond to the spectrum of the total power, fundamental and higher order mode, respectively. The spectrum of the higher-order mode is shifted in $-10 \mathrm{dBm}$ for the sake of clarity. The smoothed spectra are plotted with thick (red) lines.

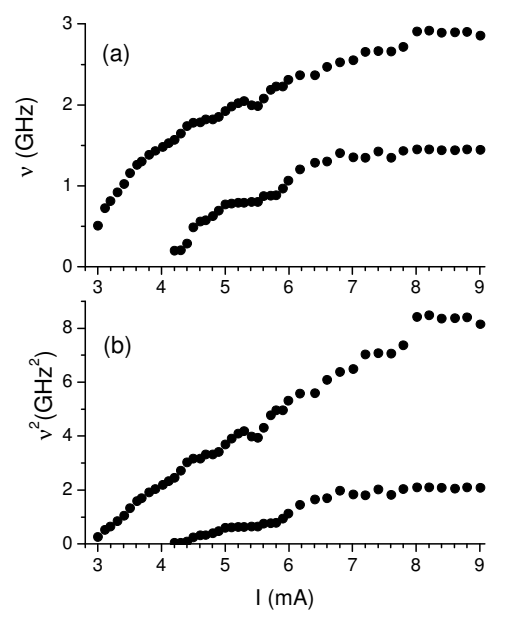

Fig. 5. (a) Frequencies and (b) Frequencies squared at which the noise spectrum of the total power is maximum versus the bias current obtained with VCSEL 1.

\section{INTENSITY NOISE SPECTRA OF A THREE-MODE VCSEL WITH POLARIZATION INSTABILITIES.}

We now analyze the current-dependence of the noise spectrum of another VCSEL (VCSEL 2) that is characterized by the appearance of a third transverse mode at large values of the bias current. While the VCSEL is emitting in the three transverse mode regime we find changes in the polarization of the modes as the current is changed. Our three-mode VCSEL has a threshold current of $3.65 \mathrm{~mA}$. Above that current value the VCSEL emits in the fundamental transverse mode (mode 1) until a higher order transverse mode (mode 2) begins to appear at $\mathrm{I}=7.5 \mathrm{~mA}$. The third transverse mode (mode 3 ) appears with significant power at $\mathrm{I}=11.5 \mathrm{~mA}$. Intensity noise spectra similar to those of the previous section are observed while the VCSEL is emitting in one or two transverse modes $(\mathrm{I}<11.5 \mathrm{~mA})$. Moreover the current-dependence of the frequencies at which the spectrum of the total power is maximum is similar to that presented in the previous section if $\mathrm{I}<8 \mathrm{~mA}$. That similar behaviour is shown in Fig. 6 in which those frequencies are plotted as a function the bias current. As in Fig. 5 two clear peaks appear at the bias current (slightly below $8 \mathrm{~mA}$ ) at which the VCSEL begins to emit in two transverse modes. Both frequencies increase when increasing the current until saturation is reached. The dependence of the frequency of the second peak on the current is such that $\mathrm{V} \sim(\mathrm{I}-$ $\left.\mathrm{I}_{\mathrm{th}, \mathrm{h}}\right)^{0.99}$. This exponent is clearly different to that obtained for VCSEL1. This difference can be explained in the following way. The threshold current of the high-order mode VCSEL2 is much larger than that of VCSEL1. Then the high-order mode is excited at values of the bias current at which saturation has been reached, as it can be seen in Fig. 6. As discussed in the previous section that situation corresponds to the range of bias currents at which the theoretical predictions fail. 


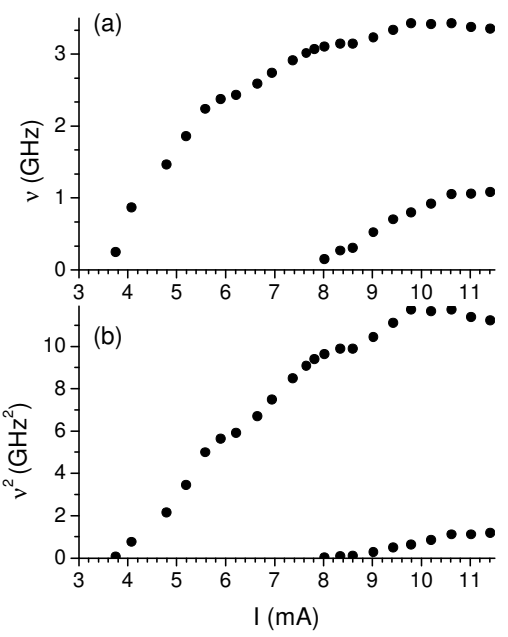

Fig. 6. (a) Frequencies and (b) Frequencies squared at which the noise spectrum of the total power is maximum versus the bias current obtained with VCSEL 2 .

We also note that the theory of Ref. [17] describes RIN spectra for arbitrary spatial coupling between two transverse modes. The theoretical results of Ref. [17] show that an 0.5 exponent is obtained if noncoupled modes are considered. The consideration of strongly coupled modes can change the exponent although it has not been studied in detail in [17].

The optical spectrum of VCSEL 2 obtained with the F-P spectrum analyzer is shown in Fig. 7 for several values of the bias current. All the frequencies in this figure are measured with respect to the frequency of the parallel polarized fundamental transverse mode (referred as mode $1_{\|}$). Single fundamental mode emission in the linear parallel polarization is illustrated in Fig. 7(a). An example of the optical spectrum in the two-mode regime is shown in Fig. 7(b) where the parallel polarized higher-order mode (referred as mode $2 \|$ ) appears at a frequency of $123.7 \mathrm{GHz}$ when $\mathrm{I}=10.88 \mathrm{~mA}$. A parallel polarized third transverse mode (mode $3 \|$ ) emits with significant power (SMSR with respect to mode $2 \|$ smaller than $10 \mathrm{~dB}$ ) when I $>11.5 \mathrm{~mA}$. The mode $3_{\|}$is the mode that appears at a $107.1 \mathrm{GHz}$ frequency in Fig. 7(c). The corresponding optical spectrum at the OSA show that each of those three modes has an associated peak that appears shifted around -40 $\mathrm{GHz}$ with respect to their frequency. Those peaks correspond to the orthogonal polarizations of the transverse modes (referred from now on with the $\perp$ subindex). Fig. 7(d) illustrates how one of those orthogonal modes (mode $2_{\perp}$ ) is excited while the corresponding parallel mode is supressed. Then a polarization switching (PS) of the mode 2 has occured at that value of the current $(\mathrm{I}=12.46 \mathrm{~mA})$. Further PS of the mode 2 appear at $\mathrm{I}=12.49$ and $12.54 \mathrm{~mA}$. Fig. 7(e) illustrates a different PS: when $\mathrm{I}=12.76 \mathrm{~mA}$ the polarization of the fundamental mode switches to the orthogonal one. The mode 1 remains in the orthogonal polarization only in a narrow current range because it switches back again at $\mathrm{I}=12.85 \mathrm{~mA}$. Fig. 7(f) shows that the optical spectrum becomes more complex at larger values of the current because there is an excitation of both polarizations of the higher order transverse modes.

Noise spectra of the total power obtained with this VCSEL are shown in Fig. 8 for several values of the bias current. In contrast with the experimental set-up of the previous section, we have now used index-matching gel at different fiber FC/PC connectors in order to minimize the reflections in those connections. The reflection peaks that appear in the noise spectra of Fig. 8 are much less pronounced than in Fig. 3 (we note that the graphs shown in Fig. 8 correspond to the direct data obtained in the RF-spectrum analyzer: no smoothing is applied). A similar behaviour to the one obtained with VCSEL 1 is shown in Figs. 8(a)-(c). In Fig. 8(a) the laser emits in a single mode and only one clear peak appears in the spectrum. In Figs. 8(b)-(c) only two parallel polarized transverse modes have significative power and a couple of broad peaks are apparent in those spectra. The situation described above changes as the bias current increases to values where PS of transverse modes begin to occur. Fig. 7(d) and Fig. 8(d) show that when the mode 2 switches its polarization a couple of additional peaks appear at 11.1 and $13.5 \mathrm{GHz}$ while the structure of the spectrum at frequencies smaller than $6 \mathrm{GHz}$ remains similar. As the bias current increases three additional peaks begin to emerge. The first two appear at 8.4 and 2.5 $\mathrm{GHz}$ while the third one develops gradually from frequencies near zero to around $145 \mathrm{MHz}$. That situation is illustrated in Fig. 8(e). The value of $2.5 \mathrm{GHz}$ corresponds to the frequency difference between the two peaks that appear at the largest frequencies. These peaks have increased and broadened when compared to the corresponding in Fig. 8(d).

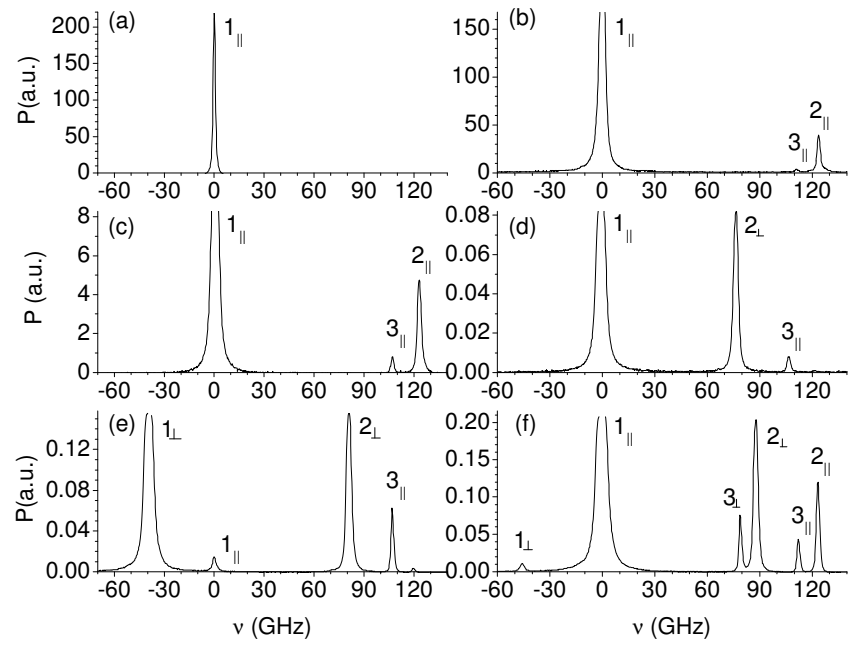

Fig. 7. Optical spectrum of the three-mode VCSEL (VCSEL 2) for several values of the bias current: (a) $7.2 \mathrm{~mA}$ (b) $10.88 \mathrm{~mA}$ (c) $12.36 \mathrm{~mA}$ (d) 12.46 $\mathrm{mA}$ (e) $12.76 \mathrm{~mA}$, and (f) $13.01 \mathrm{~mA}$.

The position of those peaks do not change significatively as the current increases and only the strength and width of those peaks change as it can be seen in Fig. 8(f). Fig. 7(f) shows that the frequency difference between the orthogonally polarized 2 and 3 modes is near $10 \mathrm{GHz}$ while the difference between the 
parallel polarized 2 and 3 modes is around $11.2 \mathrm{GHz}$. This suggests that a possible origin for the additional peaks appearing in Fig. 8(f) could correspond to the beating between the orthogonally polarized 2 and 3 modes, and the parallel polarized 2 and 3 modes, respectively.
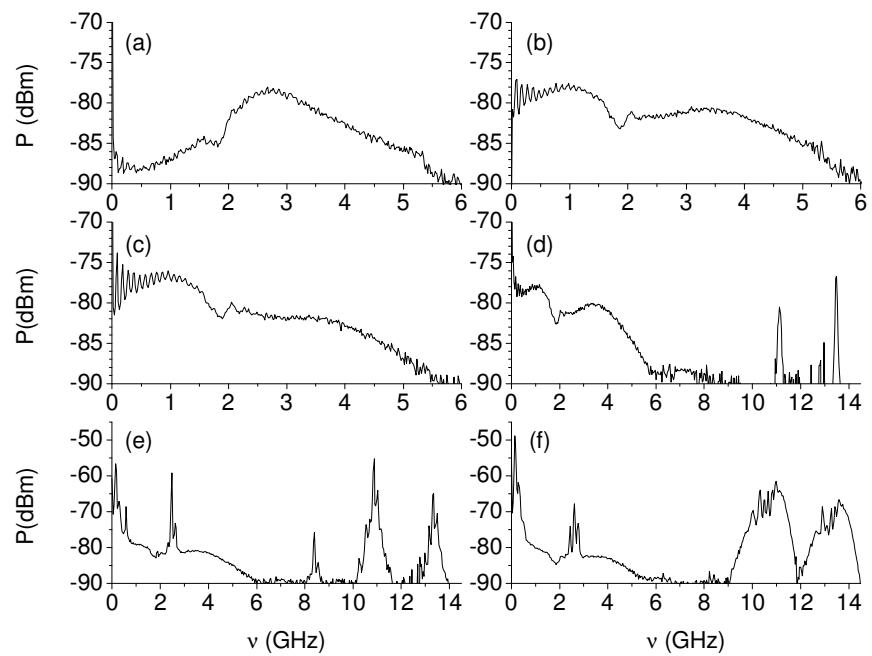

Fig. 8. RF spectrum of the three-mode VCSEL (VCSEL 2) for several values of the bias current: (a) $6.95 \mathrm{~mA}$ (b) $10.62 \mathrm{~mA}$ (c) $12.36 \mathrm{~mA}$ (d) $12.46 \mathrm{~mA}$ (e) $12.76 \mathrm{~mA}$, and (f) $13.2 \mathrm{~mA}$.

Measurements on another three-mode VCSEL (VCSEL 3) have been performed in order to confirm the hypothesis of beating between modes 2 and 3. Optical and RF spectrum are shown in Fig. 9 for a bias current value of $11.5 \mathrm{~mA}$. Fig. 9(a) shows that there is excitation of three parallel polarized transverse modes $\left(1_{\|}, 2_{\|}\right.$and $3_{\|}$modes $)$and a possible excitation of two orthogonal modes (marked as $2_{\perp}$ and $3_{\perp}$ ) that can not be resolved with our Fabry-Perot analyzer of $1 \mathrm{GHz}$ resolution. This means that the frequency separation between $2_{\perp}$ and $3_{\perp}$ modes is smaller than between $2_{\|}$and $3_{\|}$modes, like in VCSEL2. Beating between $2 \|$ and $3 \|$ modes could explain the large amplitude peak observed in Fig. 9(b) at $3.7 \mathrm{GHz}$ because the frequency difference between these modes in Fig. 9(a) is 3.9 GHz. Beating between $2_{\perp}$ and $3_{\perp}$ modes could also explain the large amplitude peak that appears at $1.42 \mathrm{GHz}$.
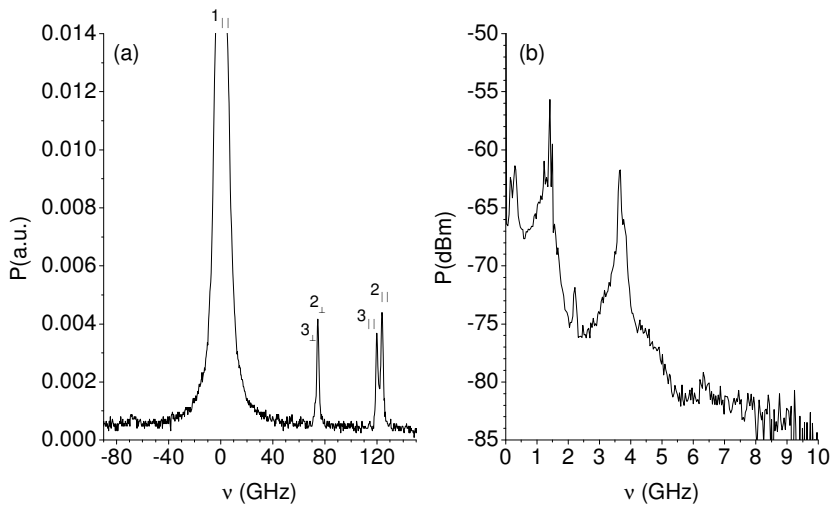

Fig. 9. (a) Optical and (b) RF spectrum of a three-mode VCSEL (VCSEL3) for a bias current of $11.5 \mathrm{~mA}$.

A reminiscence of the broad peaks obtained at lower currents (similar to those in Fig. 8(b)) is still visible in the pedestal between 3 and $5 \mathrm{GHz}$ of Fig. 9(b). Separations between $2_{\|}$and $3_{\|}$modes and between $2_{\perp}$ and $3_{\perp}$ modes are smaller than those found in VCSEL2. In this way the large amplitude peaks in the RF spectrum due to mode beating appear superimposed to the broad peaks obtained at lower currents. A low frequency peak, similar to those observed in Figs. 8(e)-(f), also appears around $0.3 \mathrm{MHz}$.

\section{DISCUSSION AND SUMMARY}

We have done spectrum calculations using the model of Ref. [19] in order to clarify the origin of the high-frequency peaks. First we have assumed that modes 1, 2 and 3 correspond to $\mathrm{LP}_{\mathrm{mn}}$ modes with different values of $\mathrm{m}$ and $\mathrm{n}$. We have considered waveguide parameters in such a way that the VCSEL emits with a wavelength near $1.56 \mu \mathrm{m}$ and with a frequency separation between the fundamental $\left(\mathrm{LP}_{01}, 1_{\|}\right.$in the experiment) and the first high-order transverse mode $\left(\mathrm{LP}_{11}\right.$, that would be $3_{\|}$in the experiment) similar to that obtained experimentally $(110 \mathrm{GHz})$. With these parameters the next high-order mode ( $\mathrm{LP}_{21}$, that would be $2_{\|}$in the experiment) appears at a frequency $150 \mathrm{GHz}$ larger than that of $\mathrm{LP}_{11}$. This value is much larger than the experimental frequency difference between $2{ }_{\|}$and $3_{\|}$. This means that the assignment of $\mathrm{LP}_{11}$ to $3_{\|}$, and of $\mathrm{LP}_{21}$ to $2_{\|}$modes has not sense. A more reasonable hypothesis is to consider that modes 2 and 3 are $\mathrm{LP}_{11}$ 's modes with different azimuthal distributions $\left(\mathrm{LP}_{11}{ }^{\mathrm{c}}\right.$ and $\mathrm{LP}_{11}{ }^{\mathrm{s}}$ with the notation of [19]). A possible reason for the splitting of $\mathrm{LP}_{11}$-mode could be an oblong aperture shape. Measurements of near or far fields would be desirable to determine whether modes 2 and 3 corresponded to $L P_{11}$ 's with different azimuthal distributions. We plan to do these measurements in the future.

In this work we have shown how the noise behavior obtained when the VCSEL is emitting in two parallel polarized transverse modes is well described with the simple theory developed in Ref. [17]. This result has been shown with the results of VCSEL 1 and VCSEL 2 with small enough values of the applied bias current. However, the measurements performed for VCSEL 2 and VCSEL 3 at large values of the current suggest that the polarization and the azimuthal dependence of transverse modes can also play an important role in determining the new structures that appear in the noise spectra. As the model of Ref. [17] fails to describe our results for VCSELs 2 and 3 at large bias currents an extension of that model to consider both linear polarizations of azimuthallydependent transverse modes would be desirable. The important role that the emission in both linear polarizations has on the RIN spectra was theoretically studied by Mulet et al. in a VCSEL emitting in the fundamental transverse mode [20]. Extensions of that model taking into account the spatial degree of freedom for considering the spatial hole burning effects that lead to emission in multiple transverse modes have already appeared in the literature [21-22] although they were not used to calculate RIN spectra. Further calculations using those models would be desirable to explain our experimental results.

To summarize, we have studied the intensity noise spectra of two types of multimode long wavelength VCSELs with different transverse and polarization modes characteristics. 
The first type corresponds to a VCSEL that emits in two transverse modes for large values of the bias current. Both modes are linearly polarized and have parallel polarizations. Two resonance peaks appear in the noise spectra of the individual modes and total power of the VCSEL in agreement with previous theoretical studies [17]. The frequencies at which both peaks appear have been measured as a function of the bias current. We have obtained a similar dependence to the one found in [17]. With the second type of VCSEL an emission in three different transverse modes for large values of the bias current is obtained. Both types of VCSELs have similar behaviours while the bias current is small. However, additional peaks appear in the noise spectra as the bias current increases in such a way that emission in three transverse modes with different polarizations appears. We believe that the polarization and the higher-order transverse mode rotational instabilities can play an important role in determining the structure of noise spectra. Further theoretical and experimental work is needed to elucidate the role played by the previous mechanisms on the noise spectra of VCSELs emitting in multiple transverse modes.

\section{ACKNOWLEDGEMENTS}

The authors acknowledge the reviewers for constructive and clarifying comments.

\section{REFERENCES}

[1] F. Koyama, "Recent advances of VCSEL Photonics", J. Lightwave Technol. Vol. 24, no. 12, pp. $4502-4513$ (2006).

[2] R. E. Freund, C. A. Bunge, N. N. Ledentsov, D. Molin, and Ch. Caspar "High-speed transmission in multi-mode fibers", J. Lightwave Technol. Vol. 28, no. 4, pp. 569-586 (2010).

[3] K. H. Hahn, M. R. Tan, Y. M. Houng, and S. Y. Tang, "Large area multitransverse mode VCSELs for modal noise reduction in multimode fibre systems", Electron. Lett., 29, pp. 1482-1483 (1993).

[4] D. Parekh, X. Zhao, W. Hofmann, M. C. Amann, L. A. Zenteno, C. J. Chang-Hasnain, "Greatly enhanced modulation response of injection locked multimode VCSELs", Opt. Exp. vol 16, no. 26, 21582 (2008).

[5] C. J. Chang-Hasnain, J.P. Harbison, G. Hasnain, A.C. von Lehmen, L.T. Florez and N.G. Stoffel, "Dynamic, polarization and transverse mode characteristics of vertical cavity surface emitting lasers", IEEE $J$ Quantum Electron., vol. 27, no. 6, pp. 1402-1409 (1991).

[6] L. G. Zei, S. Ebers, J. R. Kropp, and K. Petermann, "Noise performance of multimode VCSELs", J. Lightwave Technol. vol. 19, no. 6, pp. 884892 (2001).

[7] A. Molitor, M. Blazek, J. M. Ostermann, R. Michalzik, P. Debernardi, and W. Elsaesser, "Investigations on the intensity noise of surface grating relief VCSELs", IEEE J. Quantum Electron, vol. 46, no. 4, pp. 554-561 (2010)

[8] J. P. Hermier, A. Bramati, A. Z. Khoury, V. Josse, E. Giacobino, P. Schnitzer, R. Michalzik, and K. J. Ebeling, "Noise characteristics of oxide-confined vertical-cavity surface.emitting lasers", IEEE $J$. Quantum Electron, vol. 37, no. 2, pp. 87-91 (2001).

[9] K. H. Hahn, M. R. Tan, and S. Y. Wang, "Intensity noise of large area Vertical Cavity Surface Emitting Lasers in multimode optical fiber links", Electron. Lett. 30, pp. 139-140 (1994).

[10] L. Raddatz, I. H. White, K. H. Hahn, M. R. Tan, and S. Y. Wang, "Noise performance of multimode Vertical Cavity Surface Emitting Lasers", Electron. Lett. 30, pp. 1991-1992 (1994).

[11] D. M. Kuchta, J. Gamelin, J. D. Walker, J. Lin, K. Y. Lau, and J. S. Smith, "Relative Intensity Noise of Vertical Cavity Surface Emitting Lasers", Appl. Phys. Lett., 62, pp. 1194-1196 (1993).
[12] J. L. Vey, C. Degen, K. Auen, and W. Elsaesser, "Quantum noise and polarization properties of vertical-cavity surface-emitting lasers, Phys. Rev. A, vol. 60, pp. 3284-3295 (1999).

[13] F. Principato, S. Costa, G. Ferrante, and F. Marin, "Electrical and optical low frequency noises in multimodal vertical-cavity surfaceemitting lasers", Laser Phys. Lett., no. 10, pp. $474-479$ (2006).

[14] Y. Satuby, and M. Orenstein, "Mode-coupling effects on the smallsignal modulation of multitransverse mode vertical-cavity semiconductor lasers", IEEE J. Quantum Electron., vol. 35, no. 6, pp. 944-954 (1999).

[15] J. Y. Law, and G. P. Agrawal, "Mode partition noise in vertical cavity surface emitting lasers", IEEE Photon. Technol. Lett. vol. 9, pp. 437439 (1997).

[16] A. Valle, and L. Pesquera, "Relative intensity noise of multitransversemode vertical-cavity surface-emitting lasers", IEEE Photon. Technol. Lett. vol. 13, no. 4, pp. 272-274 (2001)

[17] A. Valle, and L. Pesquera, "Theoretical calculation of relative intensity noise of multimode vertical-cavity surface-emitting lasers", IEEE $J$. Quantum Electron, vol. 40, no. 6, pp. 597-606 (2004).

[18] A. Valle, and L. Pesquera, "Analytical calculation of transverse mode characteristics in vertical-cavity surface-emitting lasers", J. Opt. Soc. Am B, vol. 19, no. 7, pp. 1549-1557 (2002).

[19] A. Valle, "Selection and modulation of high-order transverse modes in vertical-cavity surface-emitting lasers", IEEE J. Quantum Electron., vol. 34, no. 10, pp. 1924-1932 (1998)

[20] J. Mulet, C. R. Mirasso, and M. San Miguel, "Polarization resolved intensity noise in vertical-cavity surface.emitting lasers", Phys. Rev. A, vol. 64, 023817 (2001)

[21] A. Valle, I. Gatare, K. Panajotov, M. Sciamanna, "Transverse mode switching and locking in vertical-cavity surface-emitting lasers subject to orthogonal optical injection", IEEE J. Quantum Electron., vol. 43, no. 4, pp. 322-333 (2007).

[22] J. Mulet, S. Balle, "Spatio-temporal modelling of the optical properties of VCSELs in the presence of polarization effects", IEEE J. Quantum Electron., vol. 38, no. 3, pp. 291-305 (2002).

A. Quirce received the Licenciada en Física (Ms. Sc.) degree from the Universidad de Cantabria, Spain, in 2008. She is currently working toward the $\mathrm{PhD}$ degree in Physics. Her current research interests are in the areas of dynamics of vertical-cavity surface-emitting lasers and optical injection effects in semiconductor lasers.

A. Valle was born in Reinosa, Cantabria, Spain, in 1965. He received the M. Sc. and Ph.D. degree in physics from the Universidad de Cantabria, Spain, in 1988 and 1993, respectively.

During 1994 and 1995, he was a postdoctoral fellow at the School of Electronic and Electrical Engineering at the University of Bath, England. In 1996 he joined the Instituto de Física de Cantabria (CSIC-UC). Since 1998 he has been lecturer at the Departamento de Física Moderna at the University of Cantabria, Spain. His research interests are in the areas of vertical-cavity surface-emitting lasers, noise and nonlinear dynamics of semiconductor lasers.

C. Giménez received the Licenciada en Física (Ms. Sc.) degree from the Universidad de Cantabria, Spain, in 2009.

L. Pesquera was born in Vega de Infanzones, León, Spain, in 1952. He received the M.Sc. degree in physics in 1974 from the Universidad de Valladolid, Spain. He was a postgraduate fellow at the Université de Paris VI during 1977-1980. He received the Ph.D. degree in physics in 1980 from the Universidad de Cantabria, Santander, Spain.

In 1980 he joined the Departamento de Física Moderna of the Universidad de Cantabria. Since 1991 he has been Professor of Physics at the Universidad de Cantabria. In 1995 he joined the Instituto de Física de Cantabria (CSIC$\mathrm{UC})$. His research work started in the field of stochastic processes applied to Physics and he has made contributions to the foundations of quantum physics, fluctuations in nuclear reactors, disordered systems and laser physics. $\mathrm{He}$ is currently working on the modeling of noise and nonlinear properties of semiconductor lasers and their applications to optical communication systems. 